\title{
Tri-critical behavior of the Blume Capel model on a diamond lattice
}

\author{
Jander P. Santos ${ }^{\mathrm{a}, \mathrm{b}, *}$, F.C. Sá Barreto ${ }^{\mathrm{a}, \mathrm{c}}$, D.S. Rosa ${ }^{\mathrm{d}}$ \\ a Departamento de Ciências Naturais, Universidade Federal de São João del Rei, C.P. 110, CEP 36301-160 São João del Rei, MG, Brazil \\ b Departamento de Matemática, Universidade Federal de São João del Rei, C.P. 110, CEP 36301-160 São João del Rei, MG, Brazil \\ c Emeritus Professor, Departamento de Física, Universidade Federal de Minas Gerais, C.P. 110, CEP 31270-901 Belo Horizonte, MG, Brazil \\ ${ }^{\mathrm{a}}$ Instituto de Física Teórica, Universidade Estadual Paulista, C.P. 110, CEP 01140-070 São Paulo, SP, Brazil
}

\section{A R T I C L E I N F O}

\section{Keywords:}

Blume-Capel model

Diamond lattice

Critical temperature

\begin{abstract}
A B S T R A C T
The mean field approximation results are obtained in a five-site cluster on the diamond lattice from the Bogoliubov inequality. Spin correlation identities for the Blume-Capel model on diamond lattice are derived from a five-site cluster and used to obtain an effective field approximation. The free-energy, magnetization, critical frontiers and tricritical points are obtained from the mean field approximation and the effective field approximation and are compared to those obtained by other methods. From the mean-field approximation, we also studied the unstable and metastable states besides the stable states present in the model.
\end{abstract}

\section{Introduction}

The Blume-Capel model (BC) was introduced by Blume [1] and independently by Capel [2]. This model can be applied to describe many different physical situations such as multicomponent fluids, ternary alloys, ${ }^{3} \mathrm{He}^{4} \mathrm{He}$ mixtures and various magnetic problems [3]. The Hamiltonian of the Blume-Capel model is given by

$H=-\sum_{i, j} J_{i j} S_{i} S_{j}+D \sum_{i} S_{i}^{2}$,

where $J_{i j}>0, D$ is the single ion anisotropy and the first sum is over the nearest neighbors spins on the lattice. Each $S_{i}$ is restricted by $S_{i}=\{-1,0,+1\}$.

The Blume-Capel model on rectangular and hexagonal lattices exhibits a variety of multicritical phenomena such as a phase diagram with ordered ferromagnetic $(F)$ and disordered paramagnetic $(P)$ phases separated by a transition line that changes from a continuous phase transition to a first-order phase transition at a tricritical point $\left(T_{t}\right)$. This model is also a generalization of the standard Ising model. In the limit of $D \rightarrow \infty$ one recovers the two-state Ising model with $S_{i}= \pm 1$. By increasing the value of $D$, we have a decrease of $T_{c}$. Moreover, at zero temperature, the first-order transition line ends at the point $D_{c} / J=z / 2$, where $z$ is the coordination number of the lattice $[4,5]$.

The spin-1 Blume-Capel model was studied by a variety of methods such as mean-field approximation (MFA) [1,2], effective-field approximation (EFA) [4-11], the Bethe lattice approximation [12], series expansion method (SE) [13,14], cluster variation method (CVM) $[15,16]$, Monte Carlo (MC) simulations [17-20], renormalization- group (RG) method [21,22] and rigorous inequality correlation function [23-25]. Most of these studies were done on hexagonal and rectangular lattices.

The MFA [1,2] was the first technique used to obtain an approximate solution to the Blume-Capel model. The EFA is one of the first improvements over the MFA. The EFA was used by Honmura and Kaneyoshi [26] to study the spin-1/2 Ising model and has been generalized to treat the spin-1 model [7]. This conventional EFA includes spin-spin correlations resulting from the usage of the van der Waerden relation and provides results that are superior to those obtained within the traditional MFA. The critical behavior of the Blume Emery Griffiths (BEG) spin 1 model on a diamond lattice (see Fig. 1) was studied within the framework of a finite cluster theory [28]. In this study it was used an expansion technique for the cluster identities that allows the kinematic properties of the spin operators be accounted for in a systematic way. Ekiz et al. [15,29] studied the unstable and metastable phases present in the BC and BEG models..

The aim of the present work is to study the spin-1 Blume-Capel model on the diamond lattice in a cluster of spins with five sites (see Fig. 2), to obtain the multicritical phenomena. The five-site cluster contains all the symmetries of the diamond lattice. To accomplish this objective, we present a generalization of the mean field approximation for the model of spin-1 Blume-Capel in a five-site cluster on the diamond lattice, using the Bogoliubov inequality [30-32], getting results for free-energy, phase diagrams and magnetization. To study the EFA, we derive a correlation function identity for a cluster of spins with five sites. From the correlation identities we obtain results for the magnetization, phase transitions lines and tricritical points with the

\footnotetext{
* Corresponding author at: Departamento de Matemática, Universidade Federal de São João del Rei, C.P. 110, CEP 36301-160 São João del Rei, MG, Brazil.

E-mail addresses: jander@ufsj.edu.br (J.P.Santos), fcsabarreto@gmail.com (F.C. Sá Barreto), derick@ift.unesp.br (D.S. Rosa).
} 


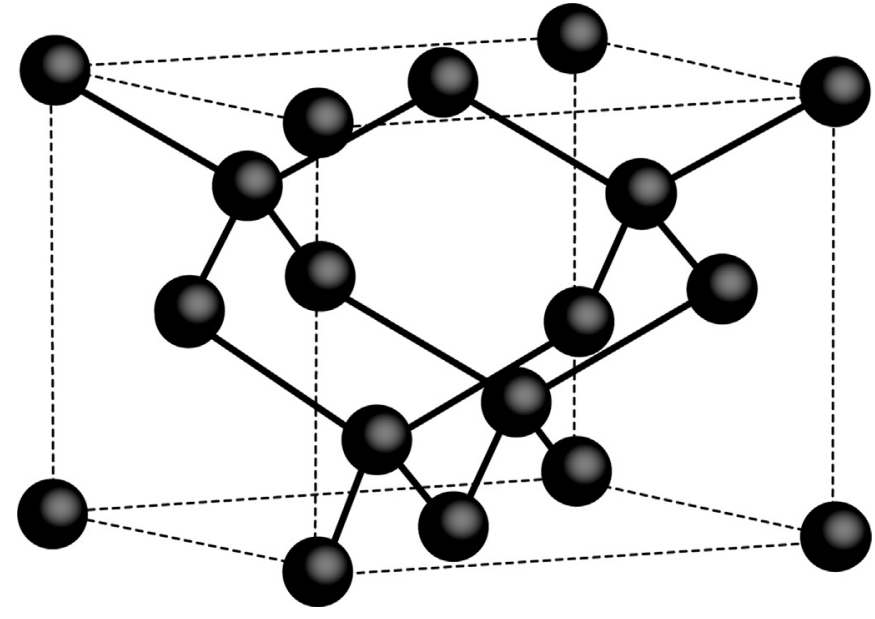

Fig. 1. Diamond lattice.

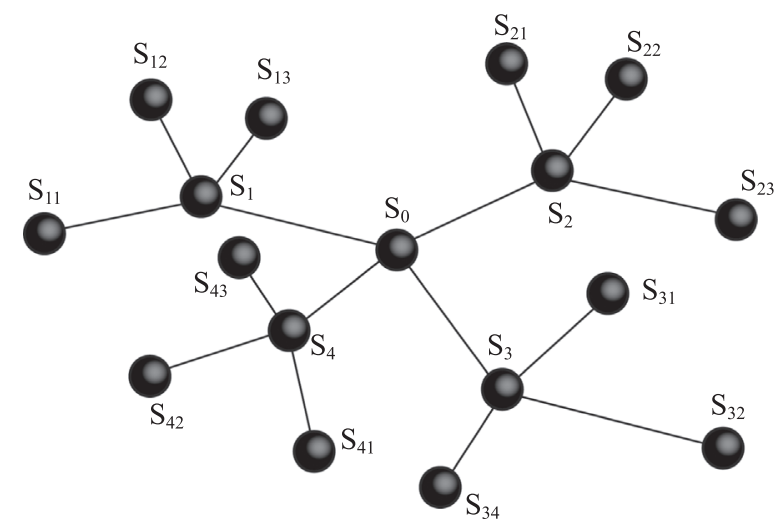

Fig. 2. Structure of five-site cluster for the diamond lattice.

application of the EFA which, as has been said previously, includes the effects of autocorrelations through the usage of the van der Waerden identities and provide results that are superior to those obtained within the MFA. In the application of the EFA a free-energy functional [4-6] was used to obtain the line of first order transitions. We remark that the results obtained improve over the one-site MFA and the one-site EFA because the five-site cluster contains more correlations than the one-site cluster. Moreover, the one-site MFA and EFA give the results for the two dimensional square lattice, which definitely are the wrong results for the diamond lattice. From the mean-field approximation, we also studied the unstable and metastable states besides the stable states present in the model..

The Section 2 is devoted to the mean field approximation, to obtain expressions for free energy and magnetization, in a five-site cluster of the spin-1 Blume-Capel model on the diamond lattice starting from the Bogoliubov inequality. In Section 3.1, we present the derivation of the correlation identities for the five-site cluster of the spin-1 Blume-Capel model on the diamond lattice, which are an extension of Callen's identity for spin 1/2 Ising model [33] and for the spin-1 Blume-Capel model for the one-site cluster [7]. The correlation identity for the spin1 Blume-Capel model obtained from the one-site cluster is suitable to study the model in hexagonal and rectangular lattices. However, for the diamond lattice this one-site correlation identity does not contain the symmetries of the lattice. In this case one has to rely on clusters which contain all the symmetries of the lattice, which is the case of the fivesite cluster, shown in Fig. 2. In Section 3.2, from these identities, derived in the previous section, we obtain the EFA and apply them to get the phase diagrams. The correlation identities can be used to derive rigorous upper bounds on critical temperatures using inequalities for the higher order correlation functions. However, this will not be done here. In Section 4, we present the numerical results and the phase diagrams as compared to other methods and in Section 5, we make the concluding remarks.

\section{Mean field approximations}

In this section we use the mean field approximation for BlumeCapel model on the diamond lattice starting from the Bogoliubov inequality [30-32], to obtain results for free-energy, phase diagrams and magnetization. The mean-field theory presumes a virtual independence of the spins variables, which is achieved by the renormalization of the actual magnetic field to the effective field taking into account the contribution from the averaged nearest-neighbor interactions. The foremost advantage of the mean-field theory lies in that it can be readily generalized to more complex lattice-statistical models as for instance the spin-1 Blume-Capel model [1,2], which may even display a greater versatility of the critical behavior including a change of continuous phase transitions to discontinuous ones at a tricritical point.

The Hamiltonian of the Blume-Capel model, is given by

$H=-J \sum_{\langle i, j\rangle}^{N z / 2} S_{i} S_{j}+D \sum_{i=1}^{N} S_{i}^{2}$,

where $N$ denotes the total number of the lattice sites and $z$ is their coordination number.

The Bogoliubov variational principle is based on a validity of the inequality [30-32]:

$G \leq \phi=G_{0}+\left\langle H-H_{0}\right\rangle_{0}$,

where $G$ and $H$ represent the true Gibbs free energy and the full Hamiltonian of a considered lattice-statistical model, whereas $G_{0}$ and $H_{0}$ represent the trial Gibbs free energy and the trial Hamiltonian of a simplified lattice-statistical model for which the relevant calculations can be performed exactly (the symbol $\langle\ldots\rangle_{0}$ denotes canonical ensemble average within the simplified model defined by the Hamiltonian $H_{0}$ ). The expression $\phi$ represents the variational Gibbs free energy, which provides an upper bound for the true Gibbs free energy $G$.

Here we present a generalization of the mean field theory for the model of spin-1 Blume-Capel in a five-site cluster on the diamond lattice, using the Bogoliubov inequality. The objective of developing the mean field theory on a higher of cluster $(n>1)$ is to maintain the symmetry of the lattice. For example the square, Kagome and diamond lattices exhibit the same coordination number $(z=4)$ and different symmetries. To accomplish this we use within the mean-field approximation a summation over the frontier sites of a cluster of five sites of the diamond lattice (see Fig. 2) and replaces an expectation value for all other spins by the mean value. Hence, it follows that the Hamiltonian of five spin is at the mean-field level, given by

$H_{5}=-\left(J S_{0} \sum_{j=1}^{4} S_{j}+J \sum_{j=1}^{4} S_{j}\left(\sum_{k=1}^{3}\left\langle S_{j k}\right\rangle\right)-D S_{0}^{2}-D \sum_{j=1}^{4} S_{j}^{2}\right)$.

$H_{5}$ is the Hamiltonian describing the site 0 and its neighbors $j=1,2,3,4$ and $S_{j k}$ are the neighbors of sites $j$.

According to this, the mean-field approximation of the spin-1 Blume-Capel in a five-site cluster on the diamond lattice (see Fig. 2) can be accomplished by the following choice of the trial Hamiltonian,

$H_{0}=-\sum_{\langle j, 0\rangle}^{N^{\prime}}\left(J S_{0} \sum_{j=1}^{4} S_{j}+\sum_{j=1}^{4} \gamma_{j} S_{j}-D S_{0}^{2}-\sum_{j=1}^{4} \eta_{j} S_{j}^{2}\right)$,

which depends on two different variational parameters $\gamma_{j}$ and $\eta_{j}$ with the physical meaning of an effective field. The $N^{\prime}$ denotes the total number of the cluster with five sites on the diamond lattice $\left(N^{\prime}=N / 5\right)$.

Eq. (5) has the physical meaning of the effective field and the effective single-ion anisotropy acting on a spin and quadrupolar 
moment, respectively, on the frontier of a cluster of five sites for the diamond lattice. With regard to this Hamiltonian (2) of the interacting many-particle system is approximated by the Hamiltonian (5) of the non-interacting system of the spin-1 particles, for which the partition function $Z_{0}$, the Gibbs free energy $G_{0}$, the magnetization $m_{0}=\left\langle S_{i}\right\rangle_{0}$ and the quadrupolar moment $q_{0}=\left\langle S_{i}^{2}\right\rangle_{0}$ can easily be calculated:

$$
\begin{aligned}
Z_{0} & =\sum_{\left\{S_{j}\right\}} \exp \left(-\beta H_{0}\right) \\
& =\prod_{j=1}^{N^{\prime}} \sum_{S_{j}= \pm 1,0} \exp \left(\beta J S_{0} \sum_{j=1}^{4} S_{j}+\beta \sum_{j=1}^{4} \gamma_{j} S_{j}-\beta D S_{0}^{2}-\beta \sum_{j=1}^{4} \eta_{j} S_{j}^{2}\right),
\end{aligned}
$$

$G_{0}=-N^{\prime} k_{B} T \ln \left(Z_{0}\right)$

$m_{0}=m_{j}=-\frac{1}{N^{\prime}} \frac{\partial G_{0}}{\partial \gamma_{j}}$

$q_{0}=q_{j}=-\frac{1}{N^{\prime}} \frac{\partial G_{0}}{\partial \eta_{j}}$

The mean value for the difference between the true and trial Hamiltonians entering the right hand-side of the Bogoliubov inequality (3), is given by,

$\left\langle H-H_{0}\right\rangle_{0}=-N^{\prime} 6 J m_{0}^{2}+N^{\prime} 4 D q_{0}+N^{\prime} m_{0} \sum_{j=1}^{4} \gamma_{j}-N^{\prime} q_{0} \sum_{j=1}^{4} \eta_{j}$,

which leads in combination with Eq. (6)-(9) to the following expression for the variational Gibbs free energy $\phi$ determining an upper bound for the true Gibbs free energy,

$\phi=-N^{\prime} k_{B} T \ln \left(Z_{0}\right)-N^{\prime} 6 J m_{0}^{2}+N^{\prime} 4 D q_{0}+N^{\prime} m_{0} \sum_{j=1}^{4} \gamma_{j}-N^{\prime} q_{0} \sum_{j=1}^{4} \eta_{j}$.

The minimum of the variational Gibbs free energy (11) with respect to both variational parameters $\gamma_{j}$ and $\eta_{j}$, leads to,

$\frac{\partial \phi}{\partial \gamma_{j}}=0 \Leftrightarrow N^{\prime}\left(\sum_{j=1}^{4} \gamma_{j}-12 J m_{0}\right) \frac{\partial m_{0}}{\partial \gamma_{j}}-N^{\prime}\left(\sum_{j=1}^{4} \eta_{j}-4 D\right) \frac{\partial q_{0}}{\partial \gamma_{j}}=0$

$\frac{\partial \phi}{\partial \eta_{j}}=0 \Leftrightarrow N^{\prime}\left(\sum_{j=1}^{4} \gamma_{j}-12 J m_{0}\right) \frac{\partial m_{0}}{\partial \eta_{j}}-N^{\prime}\left(\sum_{j=1}^{4} \eta_{j}-4 D\right) \frac{\partial q_{0}}{\partial \eta_{j}}=0$

which are simultaneously satisfied if the variational parameters are $\eta_{j}=D$ and $\gamma_{j}=3 J m_{0}$.

The mean-field calculation thus yields, after substituting the variational parameters $\gamma_{j}$ and $\eta_{j}$ satisfying the extremum condition $\eta_{j}=D$ and $\gamma_{j}=3 J m_{0}$ into Eq. (11), the following estimate of the Gibbs free energy,

$G=-N^{\prime} k_{B} T \ln \left[\exp \left(\beta J S_{0} \sum_{j=1}^{4} S_{j}+3 \beta J m \sum_{j=1}^{4} S_{j}-\beta D S_{0}^{2}-D \beta \sum_{j=1}^{4} S_{j}^{2}\right)\right]$

$+N^{\prime} 6 \mathrm{Jm}^{2}$.

We have sum explicitly over the variables $S_{0}, S_{1}, S_{2}, S_{3}$, and $S_{4}$ and we find the expressions for $G$ given by Eq. (A.3) in Appendix A. The magnetization $\left(m=m_{0}\right)$ and the quadrupolar moment $\left(q=q_{0}\right)$ are determined by the expressions Eqs. (8) and (9), and are given explicitly by Eqs. (A.1) and (A.2), respectively, in Appendix A.

To study the critical states, we get the Landau expansion of the Gibbs free energy (14), which takes the following form in a close vicinity of the critical point,

$G(m \rightarrow 0)=N^{\prime}\left(g_{0}+g_{2} m^{2}+g_{4} m^{4}+\ldots\right)$,

where $g_{0}, g_{2}$ and $g_{4}$ are given by Eqs. (A.5), (A.6) and (A.7), respectively, in Appendix A.
The necessary condition $g_{2}=0$ and $g_{4}>0$ for presence of the continuous phase transition within the Landau theory of phase transitions in concordance with the critical condition Eq. (A.1), for $(m \rightarrow 0)$. The special constraint $g_{2}=0, g_{4}=0$ in the Landau theory of phase transitions is prerequisite for an existence of tricritical point, which occurs in the spin-1 Blume-Capel model. The first-order transition line is determined from a comparison of the Gibbs free energies Eq. (A.3) of the ferromagnetic state with a non-zero spontaneous magnetization $m \neq 0$ and the disordered paramagnetic state with a zero spontaneous magnetization $m=0$ both satisfying the 'self-consistent' Eq. (A.1), for the magnetization, i.e, $G(m=0)=G(m \neq 0)$.

\section{Derivation of the correlation identity and the effective field approximation}

In this section, we present the derivation of the correlation identity and the effective field approximation for the Blume-Capel spin-1 model in the diamond lattice, to obtain the magnetization, the critical frontiers and the tricritical points.

\subsection{Derivation of the correlation identity}

In this section, we present the derivation of the correlation identities for a cluster of five spins (see Fig. 2) of the Blume-Capel spin-1 model in the diamond lattice. These correlation identities for the spin-1 Blume-Capel model in the diamond lattice are a generalization of the correlation identity obtained for the model in a cluster of one spin and are derived in an analogous manner [7,23].

We define the thermal average $\langle\ldots\rangle$ by

$\langle\cdots\rangle=\frac{\sum_{\left\{S_{i}\right\}}(\cdots) \mathrm{e}^{-\beta H}}{\sum_{\left\{S_{i}\right\}} \mathrm{e}^{-\beta H}}$,

where each $S_{i}$ is restricted by $S_{i}=\{-1,0,+1\}$. Let

$\left\langle F(\{S\}) S_{i}\right\rangle=\frac{\operatorname{Tr}\left(F(\{S\}) S_{i} \mathrm{e}^{-\beta H}\right)}{\operatorname{Tr}\left(\mathrm{e}^{-\beta H}\right)}$,

where $F(\{S\})$ is any function of $S$ different from $S_{i}$.

In what follows, we consider a cluster of five sites which retains the symmetries of the diamond lattice (see Fig. 2). We can write $H=H_{i}+H^{\prime}$, where

$H_{i}=-J S_{0} \sum_{j=1}^{4} S_{j}-J \sum_{j=1}^{4} S_{j}\left(\sum_{k=1}^{3} S_{j k}\right)-D\left(S_{0}^{2}+\sum_{j=1}^{4} S_{j}^{2}\right)$,

is the Hamiltonian describing the site $i=0$ and its neighbors $j=1,2,3,4$ and $S_{j k}$ are the neighbors of sites $j . H^{\prime}$ corresponds to the Hamiltonian of the rest of the lattice. The spin variables at different sites commute and consequently $\left[H_{i}, H^{\prime}\right]=0$,

From Eqs. (17) and (18), we get,

$\left\langle F(\{S\}) S_{i}\right\rangle=\frac{\operatorname{Tr} F(\{S\}) \mathrm{e}^{-\beta\left(H_{i}+H^{\prime}\right)} S_{i}}{\operatorname{Tr}^{-\beta\left(H_{i}+H^{\prime}\right)}}=\frac{\operatorname{Tr}^{\prime} T r_{i} F(\{S\}) \mathrm{e}^{-\beta H_{i}} S_{i} \mathrm{e}^{-\beta H^{\prime}}}{\operatorname{Tr}^{\prime} T r_{i} \mathrm{e}^{-\beta H_{i}} \mathrm{e}^{-\beta H^{\prime}}}$,

or

$\left\langle F(\{S\}) S_{i}\right\rangle=\frac{\operatorname{Tr}^{\prime} T r_{i} F(\{S\}) \mathrm{e}^{-\beta H_{i}} \mathrm{e}^{-\beta H^{\prime}} \frac{T_{i} \mathrm{e}^{-\beta H_{i}} S_{i}}{\operatorname{rri}_{i} \mathrm{e}^{-\beta H_{i}}}}{\operatorname{Tr}^{\prime} T r_{i} \mathrm{e}^{-\beta H_{i}} \mathrm{e}^{-\beta H^{\prime}}}$,

where $T r^{\prime} T r_{i}=T r . T r_{i}$ is a sum over the spins variables at sites $0,1,2,3$ and 4. Finally, we obtain,

$\left\langle F(\{S\}) S_{i}\right\rangle=\left\langle F(\{S\}) \frac{T r_{i} \mathrm{e}^{-\beta H_{i}} S_{i}}{T r_{i} \mathrm{e}^{-\beta H_{i}}}\right\rangle$,

or 
$\left\langle F(\{S\}) S_{i}\right\rangle=\left\langle F(\{S\}) \frac{\sum_{\left\{S_{0}, S_{1}, \ldots, S_{4}\right\}} S_{i} \mathrm{e}^{-\beta H_{i}}}{\sum_{\left\{S_{0}, S_{1}, \ldots, S_{4}\right\}} \mathrm{e}^{-\beta H_{i}}}\right\rangle$.

Explicitly operating the trace $T r_{i}$, we get,

$\left\langle F(\{S\}) S_{i}\right\rangle=\left.\left\langle F(\{S\}) \prod_{j=1}^{4} \prod_{k=1}^{3} \mathrm{e}^{\left(\beta J S_{j k}\right) \nabla_{j}}\right\rangle f\left(a_{1}, a_{2}, a_{3}, a_{4}\right)\right|_{(0,0,0,0)}$,

where $\nabla_{j} \equiv \frac{\partial}{\partial a_{j}}$ and $a_{j}$ are variables of the function $f$, such that $\mathrm{e}^{\alpha \nabla_{1}} f\left(a_{1}, a_{2}, a_{3}, a_{4}\right)=f\left(a_{1}+\alpha, a_{2}, a_{3}, a_{4}\right)$ and $\alpha=\beta J S_{j k}$ with similar representations for $\mathrm{e}^{\alpha \nabla_{j}} f\left(a_{1}, a_{2}, a_{3}, a_{4}\right), j=1,2,3,4$. To get the function $f\left(a_{1}, a_{2}, a_{3}, a_{4}\right)$ we consider the local Hamiltonian (18) as a function of $a_{j} \equiv \sum_{k=1}^{3} S_{j k}, \quad j=1,2,3,4$, i.e.,

$-\beta H_{i}=\beta J S_{0} \sum_{j=1}^{4} S_{j}+\beta J \sum_{j=1}^{4} S_{j} a_{j}-\beta D\left(S_{0}^{2}+\sum_{j=1}^{4} S_{j}^{2}\right)$

We sum explicitly over the variables $S_{0}, S_{1}, S_{2}, S_{3}$, and $S_{4}$ and we find the expressions for

$f\left(a_{1}, a_{2}, a_{3}, a_{4}\right)=\frac{\sum_{\left\{S_{0}, S_{1}, \ldots, S_{4}\right\}} S_{i} \mathrm{e}^{-\beta H_{i}}}{\sum_{\left\{S_{0}, S_{1}, \ldots, S_{4}\right\}} \mathrm{e}^{-\beta H_{i}}}$.

The calculations were developed by using an algebraic computation software. The final expressions are trivially obtained, but are too long to be shown here.

Using the van der Waerden relations

$S_{j}^{2 n}=S_{j}^{2} \quad$ and $\quad S_{j}^{2 n+1}=S_{j}$,

for $n=1,2,3, \ldots$, we obtain,

$\mathrm{e}^{S_{j} A}=S_{j}^{2} \cosh (A)+S_{j} \sinh (A)+1-S_{j}^{2}$,

and, applying Eq. (27) in (23), we get,

$$
\begin{aligned}
\left\langle F(\{S\}) S_{i}\right\rangle= & \left\langle F(\{S\}) \prod_{j=1}^{4} \prod_{k=1}^{3}\left[1+S_{j k} \sinh \left(\beta J \nabla_{j}\right)+S_{j k}^{2}\left(\cosh \left(\beta J \nabla_{j}\right)-1\right)\right]\right\rangle \\
& \left.f\left(a_{1}, a_{2}, a_{3}, a_{4}\right)\right|_{(0,0,0,0)} .
\end{aligned}
$$

Similarly, for the correlation functions involving the square of the spin function $S_{0}^{2}$, we obtain,

$\left\langle G(\{S\}) S_{i}^{2}\right\rangle=\left.\left\langle G(\{S\}) \prod_{j=1}^{4} \prod_{k=1}^{3} \mathrm{e}^{\left(\beta J S_{j k}\right) \nabla_{j}}\right\rangle g\left(a_{1}, a_{2}, a_{3}, a_{4}\right)\right|_{(0,0,0,0)}$,

where the function $g\left(a_{1}, a_{2}, a_{3}, a_{4}\right)$ is obtained by the same procedure used to get the function $f\left(a_{1}, a_{2}, a_{3}, a_{4}\right)$, resulting in,

$$
\begin{aligned}
\left\langle G(\{S\}) S_{i}^{2}\right\rangle= & \left\langle G(\{S\}) \prod_{j=1}^{4} \prod_{k=1}^{3}\left[1+S_{j k} \sinh \left(\beta J \nabla_{j}\right)+S_{j k}^{2}\left(\cosh \left(\beta J \nabla_{j}\right)-1\right)\right]\right\rangle \\
& \left.g\left(a_{1}, a_{2}, a_{3}, a_{4}\right)\right|_{(0,0,0,0)},
\end{aligned}
$$

where $G(\{S\})$ is any function of $S$ different from $S_{i}$.

The Eqs. (28) and (30) generalize for the diamond lattice the correlation identities for the spin-1 Blume Capel model obtained for the cluster of one spin [7,23]. As already mentioned, the one-site correlation identities give wrong results when applied to the diamond lattice because they do not contain the symmetries of this lattice. It is worth mention that these Eqs. (28) and (30) contain implicitly more correlations because they were obtained from a cluster of five spins. The Eqs. (23) and (28) and similarly (29) and (30) are exact. However, in the derivation of (28) and (30) the autocorrelations have been taken explicitly through the van der Waerden relations given by (26). This will be important in establishing the EFA from Eqs. (28) and (30). The combination of those two procedures, the use of a five-site cluster and of the van der Waerden relations, will improve the MFA and EFA results.
We use those identities in the next section to obtain the EFA results. Those results (EFA) will be compared with the others results for the Kagome and square lattices, which have the same coordination number $z=4$ as the diamond lattice, obtained by EFA and other methods, such as, renormalization group, series and Monte Carlo simulations.

As mentioned previously, the correlation identities, Eqs. (28) and (30), can be used in combination with inequalities for the n-spin correlation functions to obtain rigorous upper bounds on the critical temperature of the model in this lattice. However this will not be presented in this publication.

\subsection{Effective field approximations}

In this section we use the effective field approximation for the model to obtain the phase diagrams and magnetization. As has been said, applying the EFA to the exact relations (28) and (30), we will obtain better results from these approximation as compared to those given by the correlation identities of the one site cluster due to the fact that they were obtained from a cluster of five spins which incorporate more correlations to the formalism.

The EFA is obtained from Eqs. (28) and (30) by assuming the approximation in the spirit of the molecular field, i.e, independent variables. For the spin $S_{i}$, taking $F(\{S\})=1$, from Eq. (28) using the decoupling approximation [26], we get,

$$
\begin{aligned}
\left\langle S_{i}\right\rangle= & \prod_{j=1}^{4} \prod_{k=1}^{3}\left[1+\left\langle S_{j k}\right\rangle \sinh \left(\beta J \nabla_{j}\right)+\left\langle S_{j k}^{2}\right\rangle\left(\cosh \left(\beta J \nabla_{j}\right)-1\right)\right] . \\
& \left.f\left(a_{1}, a_{2}, a_{3}, a_{4}\right)\right|_{(0,0,0,0)} .
\end{aligned}
$$

The EFA results obtained from Eqs. (28) and (30) will improve over the five-site MFA results because we have used in the derivation of those equation the fact that $S_{j}^{2 n}=S_{j}^{2}$ and $S_{j}^{2 n+1}=S_{j}$ and therefore the autocorrelations have been taken explicitly.

So taking $F(\{S\})=1$ and $G(\{S\})=1$ in Eqs. (28) and (30) and using the definitions $\left\langle S_{i}\right\rangle=m$ and $\left\langle S_{i}^{2}\right\rangle=q$, we obtain,

$m=\left.\prod_{j=1}^{4}\left[1+m \sinh \left(\beta J \nabla_{j}\right)+q\left(\cosh \left(\beta J \nabla_{j}\right)-1\right)\right]^{3} \cdot f\left(a_{1}, a_{2}, a_{3}, a_{4}\right)\right|_{(0,0,0,0)}$,

and

$q=\left.\prod_{j=1}^{4}\left[1+m \sinh \left(\beta J \nabla_{j}\right)+q\left(\cosh \left(\beta J \nabla_{j}\right)-1\right)\right]^{3} \cdot g\left(a_{1}, a_{2}, a_{3}, a_{4}\right)\right|_{(0,0,0,0)}$.

Developing the right hand side of Eqs. (32) and (33), we get,

$m=A_{1}(q) m+A_{3}(q) m^{3}+A_{5}(q) m^{5}+A_{7}(q) m^{7}+A_{9}(q) m^{9}+A_{11}(q) m^{11}$,

and

$q=B_{0}(q)+B_{2}(q) m^{2}+B_{4}(q) m^{4}+B_{6}(q) m^{6}+B_{8}(q) m^{8}+B_{10}(q) m^{10}$

$+B_{12}(q) m^{12}$,

where the coefficients $A_{i}(q)$ and $B_{i}(q)$ are obtained in a similar manner to those shown in the references [11,27].

In the neighborhood of a continuous transition line, where $\mathrm{m}$ is small, the equations of state are conveniently expressed in the form

$m=a m+b m^{3}+\ldots$

and

$q=q_{0}+q_{1} m^{2}+\ldots$

The continuous transition line is found at $a=1$ with $b<0$ and the tricritical point occurs for $a=1$ with $b=0$. 
The first-order transition line is determined from the free energy using the same methodology proposed in other works [4-6]. We calculate the Helmholtz free energy by integrating Eqs. (34) with respect to the order parameter $m$, to obtain,

$$
\begin{aligned}
\mathcal{H}(m)= & \lambda_{1}(T, D)+\lambda_{2}(T, D)\left\{\left[A_{1}(q)-1\right] \frac{m^{2}}{2}+A_{3}(q) \frac{m^{4}}{4}+A_{5}(q) \frac{m^{6}}{6}\right. \\
& \left.+A_{7}(q) \frac{m^{8}}{8}+A_{9}(q) \frac{m^{10}}{10}+A_{11}(q) \frac{m^{12}}{12}\right\},
\end{aligned}
$$

where $\lambda_{1}(T, D)$ and $\lambda_{2}(T, D)$, functions of $T$ and $D$, are not relevant in the study of the phase transitions.

We use in Eq. (38) the Maxwell construction, that corresponds to the intersection point where the free energies of the phases are the same. In the case of the transitions between the ordered $(F)$ phase and the disordered $(P)$ phase we obtain the point of intersection, $\boldsymbol{H}(m)=\boldsymbol{H}(m=0)$, from Eqs. (38), given by,

$$
\begin{aligned}
& {\left[A_{1}(q)-1\right] \frac{m^{2}}{2}+A_{3}(q) \frac{m^{4}}{4}+A_{5}(q) \frac{m^{6}}{6}+A_{7}(q) \frac{m^{8}}{8}+A_{9}(q) \frac{m^{10}}{10}} \\
& \quad+A_{11}(q) \frac{m^{12}}{12}=0 .
\end{aligned}
$$

By simultaneously solving the three Eqs. (34), (35) and (39), we obtain the first order transition line.

The numerical results for the critical temperatures and the phase diagrams will be presented in Section 4.

\section{Numerical results and diagrams}

In Fig. 3(a), we present the magnetization $(\mathrm{m})$ as a function of $\left(k_{B} T / J\right)$ and $(D / J)$, for the Blume-Capel model on the diamond lattice for a cluster of the five sites resulting from the MFA Eq. (A.1). The first-order transitions for the stable state, limited between the dense ferromagnetic phase $(d f)$ and dense paramagnetic phase $(p f)$, are indicated by dashed lines that were derived from the solution of $G(m=0)=G(m \neq 0)$, using Eqs. (A.1) and (A.3). To obtain the first-order transitions for the metastable state, limited between the dense phase paramagnetic $(d f)$ and paramagnetic phase $(P)$, and indicated by dashed-dotted lines, we use the same methodology proposed in other works (see Refs. [15,29]), using Eqs. (A.1), (A.2) and (A.3). In Figs. 3(b), (c) and (d), we present the free energy (dotted lines) as a function of magnetization $(m)$, for different values from $D / J$ and $k_{B} T / J$. The solid lines indicate the stable state, the dashed-dotted lines indicate the metastable state and the dashed lines indicate unstable state. In Fig. 3 (b), we present the free energy as a function of magnetization for $(D / J=0)$ and $k_{B} T / J=\{0,1.0,1.5,1.8,2.0,2.3,2.477\}$. The solid line $(C D F)$ represent the stable state and the dashed line $(A D)$ represent the unstable state. In $k_{B} T / J=0.2477$ occurs a continuous phase transition. In Fig. 3(c), we present the free energy as a function of magnetization for $(D / J=-1.95)$ and $k_{B} T / J=\{0,0.6,0.7,0.749,0.81,0.85,0.90,0.929\}$. The solid line $(C E D F)$ represent the stable state, the dashed-dotted line $(A D E H)$ represent the metastable state and the dashed line $(B H)$ represent the unstable state. In $k_{B} T / J=0.749$ occurs a first-order phase transition for the stable state and in $k_{B} T / J=0.929$ occurs a first-order phase transition for the metastable state. In Fig. 3(d), we present the free energy as a function of magnetization for $(D / J=-2.10)$ and $k_{B} T / J=\{0,0.3,0.4,0.5,0.6,0.67,0.731\}$. The solid line $(A D)$ represent the stable state, the dashed-dotted line $(\mathrm{CH})$ represent the metastable state and the dashed line $(B H)$ represent the unstable state. In $k_{B} T / J=0.731$ occurs a first-order phase transition for the metastable state.

In Fig. 4, we present the phase diagrams for the Blume-Capel model on the diamond lattice for a cluster of the five sites resulting from the (a) mean field approximation and (b) effective field approximation. The phase transitions were studied as a function of $k_{B} T / J$ and $D / J$. In Fig. 4 (a) the diagram exhibits the ferromagnetic phase $(F)$ and paramagnetic phase $(P)$ separated by solid line of continuous phase transitions, the ferromagnetic phase and dense ferromagnetic phase $(d f)$ separated by dotted line, the dense ferromagnetic phase and dense paramagnetic phase $(d p)$ separated by dashed line of first-order phase transitions of the stable state and the dense paramagnetic phase and paramagnetic phase separated by dashed-dotted lines of first-order phase transitions of the metastable state. The temperature $k_{B} T_{c} / J$ for $(D=0),(D \rightarrow \infty)$ and tricritical point have the following values, 2.477, 3.644 and $C_{1}=(1.855,1.208)$, respectively. The phases transition lines $(F-P)$ and $(F-d f)$ are obtained from of the critical condition $g_{2}=0$ in Eq. (A.6). In Fig. 4 (b) the diagram exhibits the ferromagnetic phase $(F)$ and paramagnetic phase $(P)$ separated by solid line of continuous phase transitions and the ferromagnetic phase and paramagnetic phase separated by dashed line of first order phase transitions. The temperature $k_{B} T_{c} / J$ for $(D=0),(D \rightarrow \infty)$ and tricritical point have the following values, $2.123,2.982$ and $C_{2}=(1.894,0.940)$, respectively. Kerouad et al. [28] studied the Blume Emery Griffiths (BEG) spin 1 model on a diamond lattice and obtained $(1.894,0.95)$, for the EFA, to the tricritical point in the convergence to the Blume-Capel model, i.e., by considering biquadratic interactions coupling null. We could not get the unstable and metastable states, for the EFA, because we do not have explicit expressions for $\lambda_{1}(T, D)$ and $\lambda_{2}(T, D)$ of Eq. (38).

In Fig. 5, we present the magnetization for Blume-Capel model on the diamond lattice for a cluster of the five sites, resulting from the (a) MFA and (b) EFA. It is shown the magnetization $m$ as a function of temperature $k_{B} T / J$ for some values of the ration $D / J$. In Fig. 5 (a), we present the magnetization in the stable solutions (solid lines), in the metastable solutions (dashed-dotted lines) and in the unstable solutions (dashed lines), that can be observed in Fig. 3. In Fig. 5 (b), we present the magnetization in the stable solutions (solid lines). The firstorder phase transitions of the stable state are indicated by vertical lines and the first-order phase transitions of the metastable state are indicated by arrows. For some values of $D / J$ the results for the critical temperature $\left(k_{B} T_{C} / J\right)$, denoted by $\left(D / J, k_{B} T_{C} / J\right)$, are $(0,2.477)$, the tricritical point $(1.855,1.208)$, the first-order phase transitions of the unstable state $(1.92,0.882)$ and $(1.95,0.749)$ and the first-order phase transitions of the metastable state $(1.92,0.987),(1.95,0.929)$ and $(2.10,0.731)$ for the MFA and $(0,2.123)$, the tricritical point $(1.894$, $0.940)$, the first-order phase transitions of the unstable state (1.92, $0.841)$ and $(1.95,0.735)$ for the EFA.

The Fig. 6 depicts the temperature variation of the quadrupolar moment for the same values of the ration $D / J$ used in the magnetization curves of Fig. 5. Both a first-order and continuous transition are shown.

In Table 1 , we present the numerical results of $k_{B} T_{C} / J$ resulting from the of MFA and EFA, for the $(D=0)$ and for the Ising limit $(D / J \rightarrow \infty)$, obtained by this work for the diamond lattice and the results of other works for the Kagome and the square lattices. Note that the diamond, Kagome and square lattices present the same coordination number $(z=4)$.

We can see that the numerical results in Table 1 and phase diagrams (Fig. 4, Fig. 5 and Fig. 6) of the mean field approximation and effective field approximation for the Blume-Capel model on a diamond lattice presents a behavior similar to the Kagome and the square lattice, obtained by other works $[7-9,17,21]$. These results are better than the MFA and EFA results obtained from the expressions given by the one-site cluster, because it maintains the symmetry of the lattice. Note that by studying the diamond lattice from a one-site cluster the results would be the same as those for the square lattice, shown in the table.

We observe in Table 1 that the results of EFA, $k_{B} T_{c} / J=2.123$ for the $(D=0)$ and $k_{B} T_{C} / J=2.982$ for the Ising limit $(D / J \rightarrow \infty)$, are better than the MFA results, $k_{B} T_{c} / J=2.477$ for the $(D=0)$ and $k_{B} T_{c} / J=3.644$ for the Ising limit $(D / J \rightarrow \infty)$, because it contains the autocorrelation through the relation (26). 
a

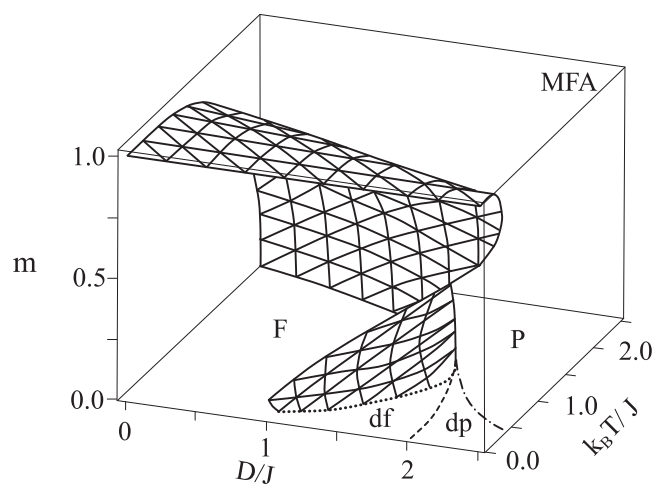

c

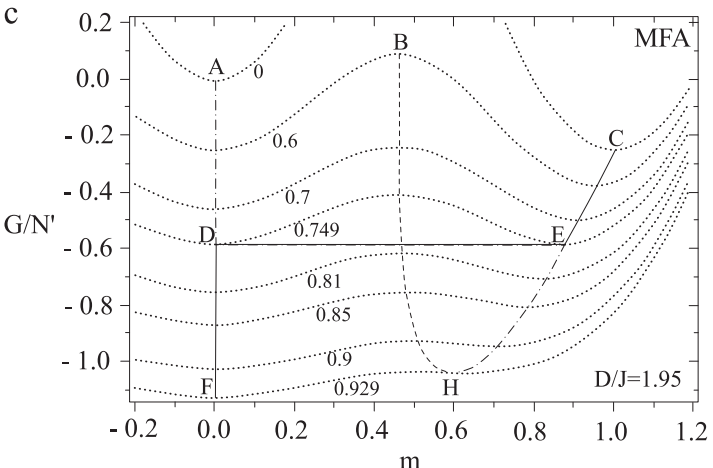

$\mathrm{b}$

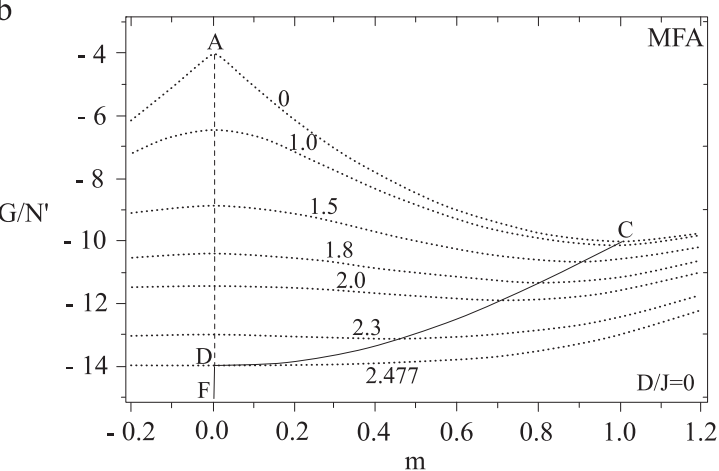

d

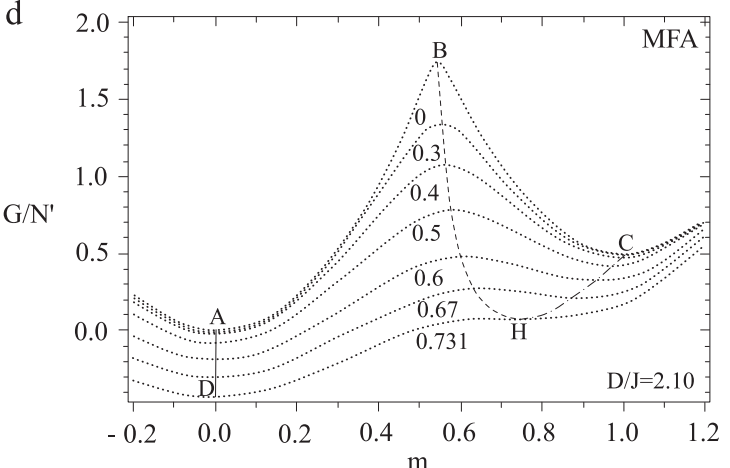

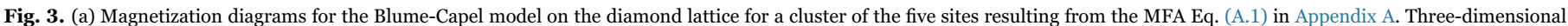

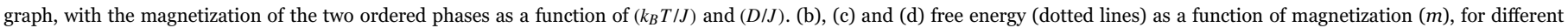
values from $D / J$ and $k_{B} T / J$. The solid lines indicate the stable state, the dashed-dotted lines indicate the metastable state and the dashed lines indicate unstable state.
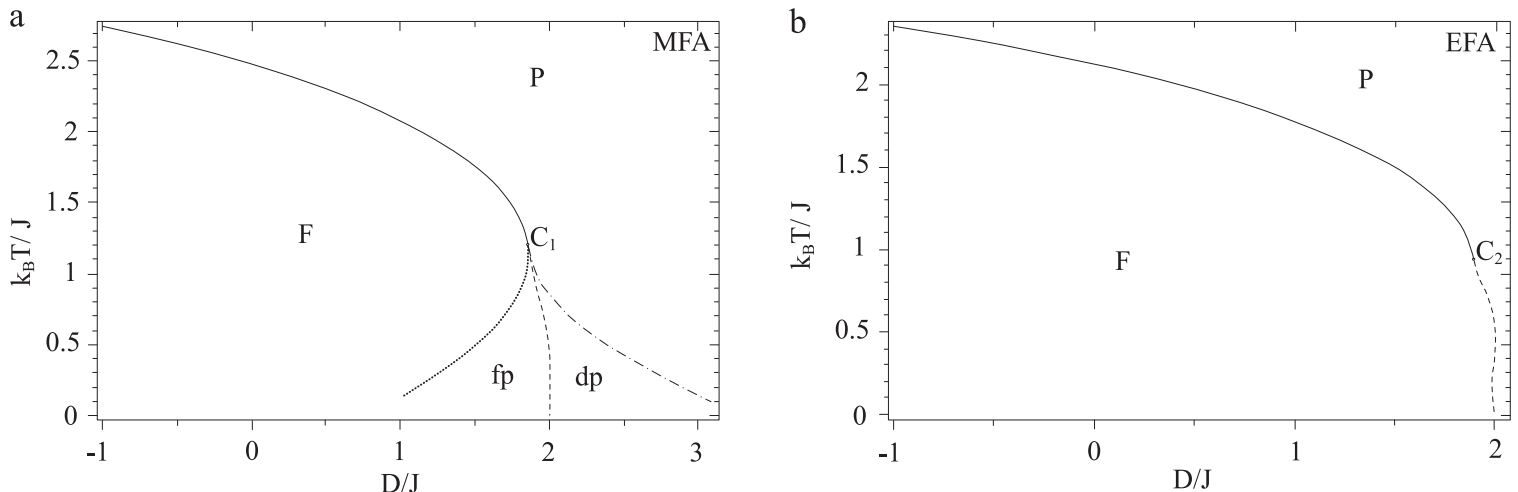

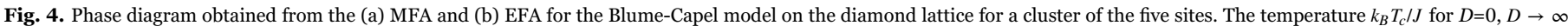

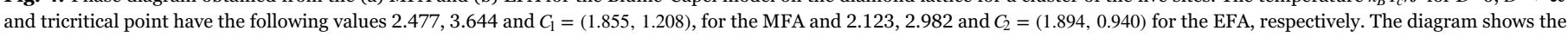
ferromagnetic $(F)$, dense ferromagnetic $(c f)$, dense paramagnetic $(c p)$, and paramagnetic $(P)$ phases.

\section{Final comments}

We have presented the mean field approximation in a five-site cluster on the diamond lattice from the generalization of Bogoliubov inequality. Also, was presented the derivation spin correlation identities for the Blume-Capel model in a five-site cluster on the diamond lattice and used in the effective field approximation. The EFA is obtained from the correlation identities from expressions (28) and (30) which contain implicitly the autocorrelation through Eq. (26) improving the results compared to the five-site MFA. These results are better than the MFA and EFA results obtained from the expressions given by the one-site cluster. Moreover, the one-site cluster correlation identity does not contain the correct symmetry of the diamond lattice. Within these approximations we obtained results for free-energy, phase diagrams and magnetization. The results obtained in the limits $D=0$, the Ising limit $(D / J \rightarrow \infty)$ and tricritical point on a diamond lattice were compared with the results for the Kagome and square latices obtained by other works. We believe that the present formalism of MFA and EFA applied to a multispin-site cluster can be extended to more complicated model systems, such as spin models in the pyrochlore lattice. This should be done in a future work.

\section{Acknowledgments}

D.S.R. acknowledges financial support from CNPq/Brazil (Project No. 147716/2014.4).

\section{Appendix A. Expressions to results of the mean field approximation}

In this section, we give an explicit representation formula for $m, q$, $G, g_{0}, g_{2}$ and $g_{4}$, obtained in Section 2. A straightforward computation leads to the expressions, 

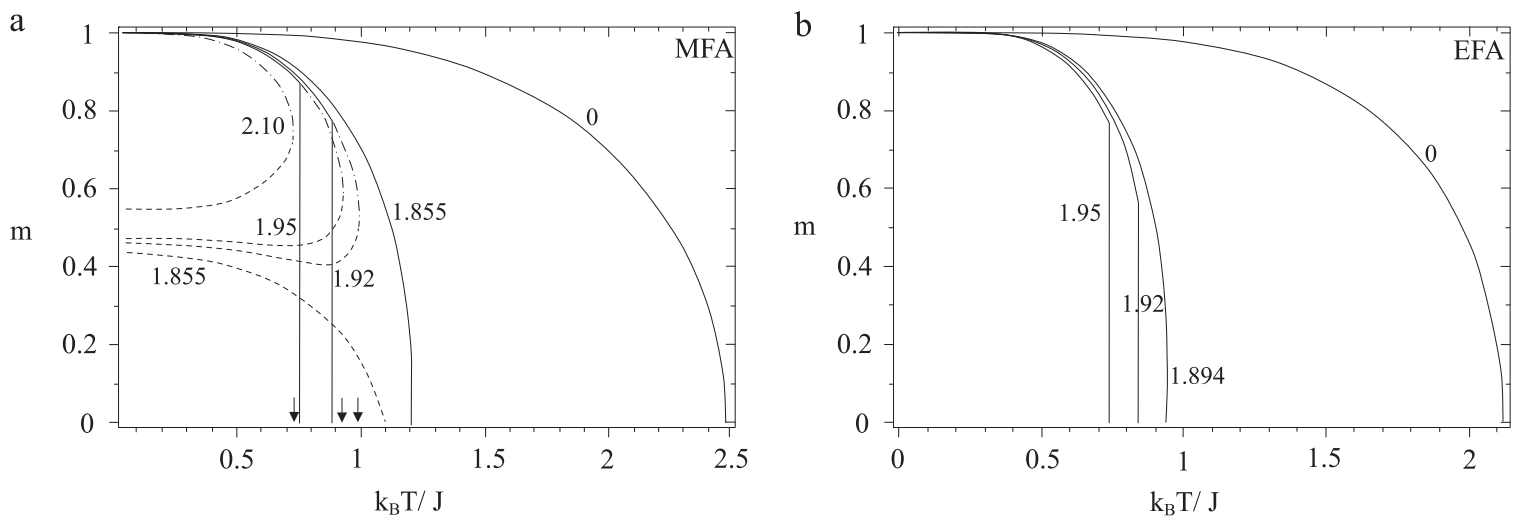

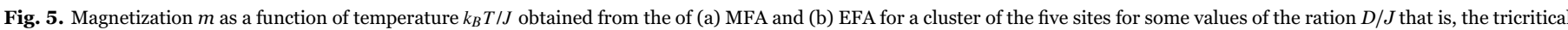

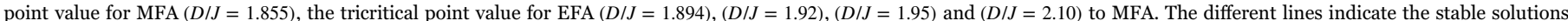

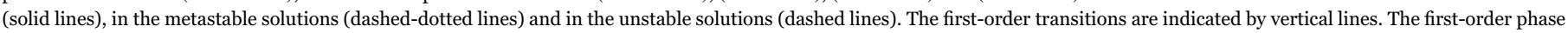
transitions of the stable state are indicated by vertical lines and the first-order phase transitions of the metastable state are indicated by arrows.
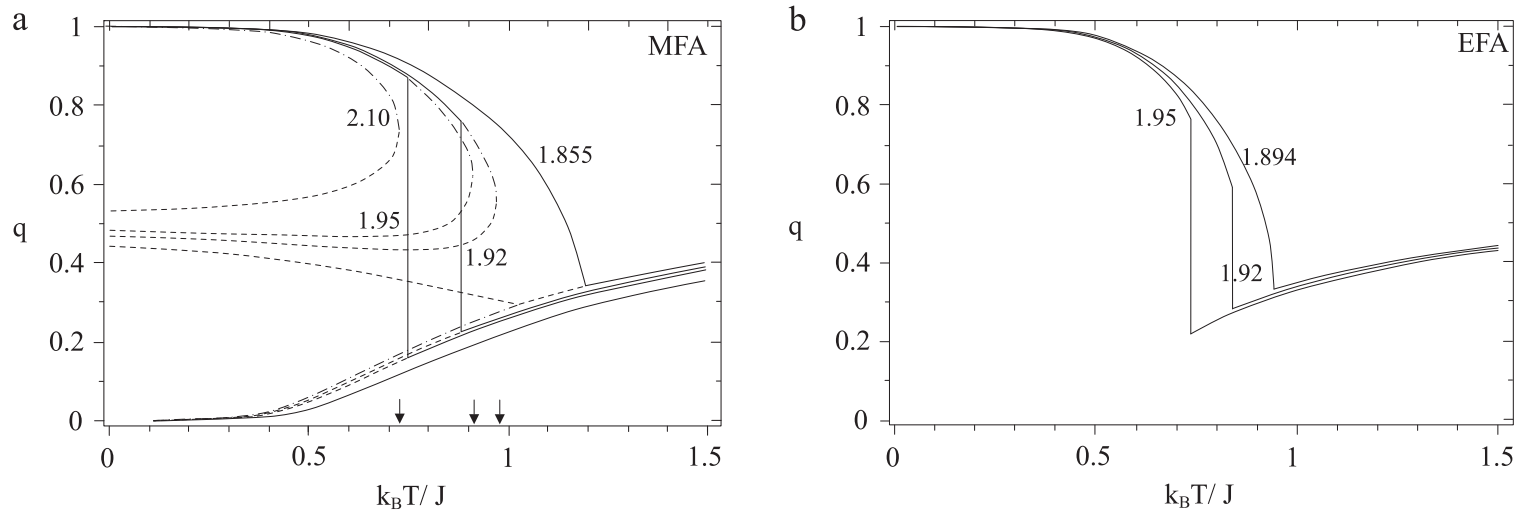

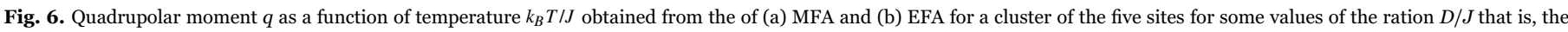

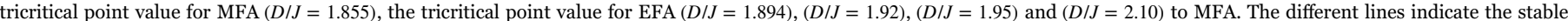

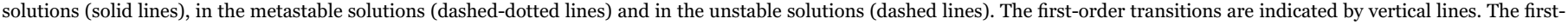
order phase transitions of the stable state are indicated by vertical lines and the first-order phase transitions of the metastable state are indicated by arrows.

$$
\begin{aligned}
m= & {\left[2 \mathrm{e}^{2 \beta(2 D+3 J m)}+3 \mathrm{e}^{2 \beta(D+3 J m)}-\mathrm{e}^{\beta(-4 J+5 D-12 J m)}+3 \mathrm{e}^{\beta(2 J+3 D+6 J m)}\right.} \\
& +\mathrm{e}^{\beta(J+2 D+3 J m)}+3 \mathrm{e}^{3 \beta(D+J m)}+\mathrm{e}^{4 \beta(D+3 J m)}-3 \mathrm{e}^{\beta(2 J+3 D-6 J m)} \\
& -3 \mathrm{e}^{3 \beta(D-3 J m)}+3 \mathrm{e}^{3 \beta(D+3 J m)}+2 \mathrm{e}^{\beta(2 J+5 D+6 J m)}+\mathrm{e}^{\beta(4 J+5 D+12 J m)} \\
& +3 \mathrm{e}^{\beta(-J+4 D+3 J m)}+3 \mathrm{e}^{\beta(3 J+4 D+9 J m)}-\mathrm{e}^{\beta(D-3 J m)}-3 \mathrm{e}^{\beta(-J+4 D-3 J m)} \\
& -\mathrm{e}^{\beta(J+2 D-3 J m)}+3 \mathrm{e}^{\beta(J+4 D+3 J m)}+\mathrm{e}^{\beta(-4 J+5 D+12 J m)}-3 \mathrm{e}^{\beta(-3 J+4 D-9 J m)} \\
& -3 \mathrm{e}^{2 \beta(D-3 J m)}-3 \mathrm{e}^{\beta(J+4 D-3 J m)}-3 \mathrm{e}^{\beta(3 J+4 D-9 J m)}+3 \mathrm{e}^{\beta(-3 J+4 D+9 J m)} \\
& -3 \mathrm{e}^{\beta(-2 J+3 D-6 J m)}-\mathrm{e}^{\beta(4 J+5 D-12 J m)}+\mathrm{e}^{\beta(D+3 J m)}+3 \mathrm{e}^{\beta(-2 J+3 D+6 J m)} \\
& -3 \mathrm{e}^{3 \beta(D-J m)}-2 \mathrm{e}^{\beta(-2 J+5 D-6 J m)}+2 \mathrm{e}^{\beta(-2 J+5 D+6 J m)}-2 \mathrm{e}^{2 \beta(2 D-3 J m)} \\
& \left.-2 \mathrm{e}^{\beta(2 J+5 D-6 J m)}-\mathrm{e}^{4 \beta(D-3 J m)}-\mathrm{e}^{\beta(-J+2 D-3 J m)}+\mathrm{e}^{\beta(-J+2 D+3 J m)}\right] / Z_{m}
\end{aligned}
$$

\footnotetext{
$q=\left[\mathrm{e}^{\beta(D-3 J m)}+9 \mathrm{e}^{\beta(-J+4 D-3 J m)}+\mathrm{e}^{\beta(J+2 D-3 J m)}+\mathrm{e}^{\beta(4 J+5 D+12 J m)}\right.$

$+9 \mathrm{e}^{\beta(-J+4 D+3 J m)}+3 \mathrm{e}^{\beta(3 J+4 D+9 J m)}+4 \mathrm{e}^{\beta(-2 J+5 D-6 J m)}+9 \mathrm{e}^{3 \beta(D-J m)}$ $+\mathrm{e}^{\beta(4 J+5 D-12 J m)}+\mathrm{e}^{\beta(D+3 J m)}+3 \mathrm{e}^{\beta(-2 J+3 D+6 J m)}+3 \mathrm{e}^{\beta(3 J+4 D-9 J m)}$$$
+3 \mathrm{e}^{\beta(-3 J+4 D+9 J m)}+3 \mathrm{e}^{\beta(-2 J+3 D-6 J m)}+3 \mathrm{e}^{2 \beta(D-3 J m)}+9 \mathrm{e}^{\beta(J+4 D-3 J m)}
$$$$
+9 \mathrm{e}^{\beta(J+4 D+3 J m)}+\mathrm{e}^{\beta(-4 J+5 D+12 J m)}+3 \mathrm{e}^{\beta(-3 J+4 D-9 J m)}+6 \mathrm{e}^{4 \beta(D)}
$$$$
+6 \mathrm{e}^{2 \beta(D)}+12 \mathrm{e}^{5 \beta(D)}+12 \mathrm{e}^{3 \beta(D)}+\mathrm{e}^{\beta(-J+2 D-3 J m)}+\mathrm{e}^{\beta(-J+2 D+3 J m)}
$$$$
+4 \mathrm{e}^{\beta(2 J+5 D-6 J m)}+\mathrm{e}^{4 \beta(D-3 J m)}+4 \mathrm{e}^{\beta(-2 J+5 D+6 J m)}+4 \mathrm{e}^{2 \beta(2 D-3 J m)}
$$$$
+4 \mathrm{e}^{2 \beta(2 D+3 J m)}+9 \mathrm{e}^{3 \beta(D+J m)}+\mathrm{e}^{4 \beta(D+3 J m)}+\mathrm{e}^{\beta(J+2 D+3 J m)}+3 \mathrm{e}^{2 \beta(D+3 J m)}
$$$$
+\mathrm{e}^{\beta(-4 J+5 D-12 J m)}+3 \mathrm{e}^{\beta(2 J+3 D+6 J m)}+3 \mathrm{e}^{\beta(2 J+3 D-6 J m)}+3 \mathrm{e}^{3 \beta(D-3 J m)}
$$$$
\left.+3 \mathrm{e}^{3 \beta(D+3 J m)}+4 \mathrm{e}^{\beta(2 J+5 D+6 J m)}\right] / Z_{m}
$$$$
G=-N^{\prime} k_{B} T \ln \left(Z_{m}\right)+N^{\prime} 6 J m^{2}
$$

$$
\begin{aligned}
Z_{m}= & {\left[1+4 \mathrm{e}^{\beta(D-3 J m)}+12 \mathrm{e}^{\beta(-J+4 D-3 J m)}+4 \mathrm{e}^{\beta(J+2 D-3 J m)}+\mathrm{e}^{\beta(4 J+5 D+12 J m)}\right.} \\
& +12 \mathrm{e}^{\beta(-J+4 D+3 J m)}+4 \mathrm{e}^{\beta(3 J+4 D+9 J m)}+12 \mathrm{e}^{3 \beta(D-J m)}+4 \mathrm{e}^{\beta(-2 J+5 D-6 J m)} \\
& +\mathrm{e}^{\beta(4 J+5 D-12 J m)}+4 \mathrm{e}^{\beta(D+3 J m)}+6 \mathrm{e}^{\beta(-2 J+3 D+6 J m)}+4 \mathrm{e}^{\beta(3 J+4 D-9 J m)} \\
& +4 \mathrm{e}^{\beta(-3 J+4 D+9 J m)}+6 \mathrm{e}^{\beta(-2 J+3 D-6 J m)}+6 \mathrm{e}^{2 \beta(D-3 J m)}+12 \mathrm{e}^{\beta(J+4 D-3 J m)} \\
& +12 \mathrm{e}^{\beta(J+4 D+3 J m)}+\mathrm{e}^{\beta(-4 J+5 D+12 J m)}+4 \mathrm{e}^{\beta(-3 J+4 D-9 J m)}+6 \mathrm{e}^{4 \beta(D)} \\
& +12 \mathrm{e}^{2 \beta(D)}+12 \mathrm{e}^{5 \beta(D)}+24 \mathrm{e}^{3 \beta(D)}+4 \mathrm{e}^{\beta(-J+2 D-3 J m)}+4 \mathrm{e}^{\beta(-J+2 D+3 J m)} \\
& +2 \mathrm{e}^{\beta(D)}+4 \mathrm{e}^{\beta(2 J+5 D-6 J m)}+\mathrm{e}^{4 \beta(D-3 J m)}+4 \mathrm{e}^{\beta(-2 J+5 D+6 J m)} \\
& +4 \mathrm{e}^{2 \beta(2 D-3 J m)}+4 \mathrm{e}^{2 \beta(2 D+3 J m)}+12 \mathrm{e}^{3 \beta(D+J m)}+\mathrm{e}^{4 \beta(D+3 J m)} \\
& +4 \mathrm{e}^{\beta(J+2 D+3 J m)}+6 \mathrm{e}^{2 \beta(D+3 J m)}+\mathrm{e}^{\beta(-4 J+5 D-12 J m)}+6 \mathrm{e}^{\beta(2 J+3 D+6 J m)} \\
& \left.+6 \mathrm{e}^{\beta(2 J+3 D-6 J m)}+4 \mathrm{e}^{3 \beta(D-3 J m)}+4 \mathrm{e}^{3 \beta(D+3 J m)}+4 \mathrm{e}^{\beta(2 J+5 D+6 J m)}\right]
\end{aligned}
$$

$$
\begin{aligned}
g_{0}= & -k_{B} T \ln \left(1+10 \mathrm{e}^{\beta(D)}+56 \mathrm{e}^{3 \beta(D)}+24 \mathrm{e}^{2 \beta(D)}+12 \mathrm{e}^{5 \beta(D)}+16 \mathrm{e}^{4 \beta(D)}\right. \\
& +2 \mathrm{e}^{\beta(4 J+5 D)}+8 \mathrm{e}^{\beta(J+2 D)}+2 \mathrm{e}^{\beta(-4 J+5 D)}+8 \mathrm{e}^{\beta(-2 J+5 D)}+12 \mathrm{e}^{\beta(2 J+3 D)} \\
& +8 \mathrm{e}^{\beta(-J+2 D)}+24 \mathrm{e}^{\beta(J+4 D)}+8 \mathrm{e}^{\beta(-3 J+4 D)}+24 \mathrm{e}^{\beta(-J+4 D)}+8 \mathrm{e}^{\beta(3 J+4 D)} \\
& \left.+8 \mathrm{e}^{\beta(2 J+5 D)}+12 \mathrm{e}^{\beta(-2 J+3 D)}\right)
\end{aligned}
$$


Table 1

Estimates for the critical temperature $k_{B} T_{c} / J$ in the Kagome, the square and the diamond lattices.

\begin{tabular}{|c|c|c|c|c|c|c|}
\hline & Kagome lattice (Five-site cluster) & & Square lattice (One-site cluster) & & Diamond lattice (Present work) & \\
\hline & $D=0$ & $\begin{array}{l}D \rightarrow \infty \\
\text { (Ising limit) }\end{array}$ & $D=0$ & $\begin{array}{l}D \rightarrow \infty \\
\text { (Ising limit) }\end{array}$ & $D=0$ & $\begin{array}{l}D \rightarrow \infty \\
\text { (Ising limit) }\end{array}$ \\
\hline MFA & $2.271[11]$ & $3.257[11]$ & $2.666[1,2]$ & $4[1,2]$ & 2.477 & 3.644 \\
\hline EFA & $1.983[11]$ & $2.742[11]$ & $2.188[7]$ & $3.088[7]$ & 2.123 & 2.982 \\
\hline IEFA & - & - & $1.9643[8]$ & - & - & - \\
\hline MC & - & - & $1.695[17]$ & - & - & $2.700[36]$ \\
\hline RG & - & - & $2.128[21]$ & $2.884[21]$ & - & - \\
\hline Exact & - & $2.1436[34]$ & - & $2.269[35]$ & - & - \\
\hline
\end{tabular}

$$
\begin{aligned}
g_{2}= & -6 J\left(-1-8 \mathrm{e}^{\beta(-J+2 D)}-24 \mathrm{e}^{\beta(-J+4 D)}+36 \beta J \mathrm{e}^{\beta(-2 J+3 D)}\right. \\
& +24 \beta J \mathrm{e}^{\beta(4 J+5 D)}+36 \beta J \mathrm{e}^{\beta(2 J+3 D)}+24 \beta J \mathrm{e}^{\beta(-4 J+5 D)}+18 \beta J \mathrm{e}^{\beta(J+4 D)} \\
& +54 \beta J \mathrm{e}^{\beta(3 J+4 D)}+24 \beta J \mathrm{e}^{\beta(2 J+5 D)}+6 \beta J \mathrm{e}^{\beta(-J+2 D)}+24 \beta J \mathrm{e}^{\beta(-2 J+5 D)} \\
& +18 \beta J \mathrm{e}^{\beta(-J+4 D)}+54 \beta J \mathrm{e}^{\beta(-3 J+4 D)}+6 \beta J \mathrm{e}^{\beta(J+2 D)}-12 \mathrm{e}^{\beta(-2 J+3 D)} \\
& -8 \mathrm{e}^{\beta(2 J+5 D)}-24 \mathrm{e}^{\beta(J+4 D)}-56 \mathrm{e}^{3 \beta(D)}-24 \mathrm{e}^{2 \beta(D)}-16 \mathrm{e}^{4 \beta(D)}-12 \mathrm{e}^{5 \beta(D)} \\
& -8 \mathrm{e}^{\beta(-2 J+5 D)}-8 \mathrm{e}^{\beta(-3 J+4 D)}-8 \mathrm{e}^{\beta(3 J+4 D)}+6 \beta J \mathrm{e}^{\beta(D)}-10 \mathrm{e}^{\beta(D)} \\
& -8 \mathrm{e}^{\beta(J+2 D)}-2 \mathrm{e}^{\beta(4 J+5 D)}-2 \mathrm{e}^{\beta(-4 J+5 D)}-12 \mathrm{e}^{\beta(2 J+3 D)}+72 \beta J \mathrm{e}^{3 \beta(D)} \\
& \left.+48 \beta J \mathrm{e}^{4 \beta(D)}+36 \beta J \mathrm{e}^{2 \beta(D)}\right) /\left(1+10 \mathrm{e}^{\beta(D)}+24 \mathrm{e}^{\beta(J+4 D)}+8 \mathrm{e}^{\beta(J+2 D)}\right. \\
& +12 \mathrm{e}^{\beta(2 J+3 D)}+8 \mathrm{e}^{\beta(-3 J+4 D)}+8 \mathrm{e}^{\beta(-2 J+5 D)}+56 \mathrm{e}^{3 \beta(D)}+24 \mathrm{e}^{2 \beta(D)} \\
& +16 \mathrm{e}^{4 \beta(D)}+12 \mathrm{e}^{5 \beta(D)}+8 \mathrm{e}^{\beta(-J+2 D)}+12 \mathrm{e}^{\beta(-2 J+3 D)}+8 \mathrm{e}^{\beta(3 J+4 D)} \\
& \left.+2 \mathrm{e}^{\beta(-4 J+5 D)}+24 \mathrm{e}^{\beta(-J+4 D)}+8 \mathrm{e}^{\beta(2 J+5 D)}+2 \mathrm{e}^{\beta(4 J+5 D)}\right)
\end{aligned}
$$$$
g_{4}=-\beta J^{2}\left[2 7 \beta ^ { 2 } J ^ { 2 } \left(64 \mathrm{e}^{\beta(4 J-5 D)}+80 \mathrm{e}^{-4 \beta D}+24 \mathrm{e}^{-2 \beta D}+81 \mathrm{e}^{\beta(-3 J-4 D)}\right.\right.
$$$$
+\mathrm{e}^{\beta(J-2 D)}+84 \mathrm{e}^{-3 \beta D}+\mathrm{e}^{-\beta D}+3 \mathrm{e}^{\beta(J-4 D)}+81 \mathrm{e}^{\beta(3 J-4 D]}+24 \mathrm{e}^{\beta(2 J-3 D)}
$$$$
+64 \mathrm{e}^{\beta(-4 J-5 D)}+\mathrm{e}^{\beta(-J-2 D)}+16 \mathrm{e}^{\beta(2 J-5 D)}+3 \mathrm{e}^{\beta(-J-4 D)}+16 \mathrm{e}^{\beta(-2 J-5 D)}
$$$$
\left.+24 \mathrm{e}^{\beta(-2 J-3 D)}\right)\left(1+2 \mathrm{e}^{\beta(4 J-5 D)}+8 \mathrm{e}^{\beta(J-2 D)}+8 \mathrm{e}^{\beta(-3 J-4 D)}\right.
$$$$
+2 \mathrm{e}^{\beta(-4 J-5 D)}+24 \mathrm{e}^{\beta(J-4 D)}+8 \mathrm{e}^{\beta(3 J-4 D)}+12 \mathrm{e}^{\beta(2 J-3 D)}+8 \mathrm{e}^{\beta(-J-2 D)}
$$$$
+8 \mathrm{e}^{\beta(2 J-5 D)}+8 \mathrm{e}^{\beta(-3 J-4 D)}+12 \mathrm{e}^{\beta(-2 J-3 D)}+24 \mathrm{e}^{\beta(-J-4 D)}+8 \mathrm{e}^{\beta(-2 J-5 D)}
$$$$
\left.+10 \mathrm{e}^{-\beta D}+56 \mathrm{e}^{-3 \beta D}+24 \mathrm{e}^{-2 \beta D}+12 \mathrm{e}^{-5 \beta D}\right)
$$$$
-18\left(\left(4 \mathrm{e}^{\beta(4 J-5 D)}+6 \mathrm{e}^{-2 \beta D}+8 \mathrm{e}^{-4 \beta D}+9 \mathrm{e}^{\beta(-3 J-4 D)}+\mathrm{e}^{\beta(J-2 D)}\right.\right.
$$$$
+3 \mathrm{e}^{\beta(-J-4 D)}+\mathrm{e}^{-\beta D}+3 \mathrm{e}^{\beta(J-4 D)}+9 \mathrm{e}^{\beta(3 J-4 D)}+6 \mathrm{e}^{\beta(2 J-3 D)}
$$$$
+4 \mathrm{e}^{\beta(-4 J-5 D)}+\mathrm{e}^{\beta(-J-2 D)}+4 \mathrm{e}^{\beta(2 J-5 D)}+4 \mathrm{e}^{\beta(-2 J-5 D)}+12 \mathrm{e}^{-3 \beta D}
$$$$
\left.+6 \mathrm{e}^{\beta(-2 J-3 D)}\right)\left(3 6 \beta ^ { 2 } J ^ { 2 } \left(4 \mathrm{e}^{\beta(4 J-5 D)}+6 \mathrm{e}^{-2 \beta D}+8 \mathrm{e}^{-4 \beta D}+9 \mathrm{e}^{\beta(-3 J-4 D)}\right.\right.
$$$$
+\mathrm{e}^{\beta(J-2 D)}+3 \mathrm{e}^{\beta(-J-4 D)}+\mathrm{e}^{-\beta D}+3 \mathrm{e}^{\beta(J-4 D)}+9 \mathrm{e}^{\beta(3 J-4 D)}+6 \mathrm{e}^{\beta(2 J-3 D)}
$$$$
+4 \mathrm{e}^{\beta(-4 J-5 D)}+\mathrm{e}^{\beta(-J-2 D)}+4 \mathrm{e}^{\beta(2 J-5 D)}
$$$$
\left.\left.\left.\left.+4 \mathrm{e}^{\beta(-2 J-5 D)}+12 \mathrm{e}^{-3 \beta D}+6 \mathrm{e}^{\beta(-2 J-3 D)}\right)\right)\right)\right) /
$$$$
\left[1+2 \mathrm{e}^{\beta(4 J-5 D)}+8 \mathrm{e}^{\beta(J-2 D)}+8 \mathrm{e}^{\beta(-3 J-4 D)}+24 \mathrm{e}^{\beta(-J-4 D)}\right.
$$$$
+2 \mathrm{e}^{\beta(-4 J-5 D)}+24 \mathrm{e}^{\beta(J-4 D)}+8 \mathrm{e}^{\beta(3 J-4 D)}+12 \mathrm{e}^{\beta(2 J-3 D)}
$$$$
+8 \mathrm{e}^{\beta(-J-2 D)}+8 \mathrm{e}^{\beta(2 J-5 D)}+12 \mathrm{e}^{\beta(-2 J-3 D)}+8 \mathrm{e}^{\beta(-2 J-5 D)}
$$$$
\left.+10 \mathrm{e}^{-\beta D}+56 \mathrm{e}^{-3 \beta D}+24 \mathrm{e}^{-2 \beta D}+12 \mathrm{e}^{-5 \beta D}+16 \mathrm{e}^{-4 \beta D}\right]^{2}
$$

\section{References}

[1] M. Blume, Phys. Rev. 141 (1966) 517.

[2] H.W. Capel, Physica 32 (1966) 966.

[3] I..D. Lawrie, S. Sarback, C. Domb, J..L. Lebowitz (Eds.), Phase Transitions and Critical Phenomena 9, Academic Press, London, 1988.

[4] E. Costabile, M.A. Amazonas, J..R. Viana, J..R. Sousa, Phys. Lett. A 376 (2012) 22.

[5] E. Costabile, J..R. Viana, J..R. Sousa, J..A. Plascak, Physica A 393 (2014) 297.

[6] J..P. Santos, F..C. SáBarreto, J. Magn. Magn. Mater. 401 (2016) 724.

[7] A..F. Siqueira, I..P. Fittipaldi, Physica A 138 (1986) 592.

[8] Y. Yuksel, U. Akinsi, H. Polat, Phys. Scr. 79 (2009) 1.

[9] H. Polat, U. Akinci, I. Sokmen, Stat. Sol. 240 (2003) 189.

[10] T. Kaneyoshi, J. Phys. Soc. Jpn. 56 (1987) 933.

[11] J..P. Santos, F..C. SáBarreto, Physica A 421 (2015) 548.

[12] Y. Tanaka, N. Uryu, J. Phys. Soc. Jpn. 50 (1981) 1140.

[13] J..G. Brankov, Przystawa, E. Praveczki, J. Phys. C 5 (1972) 3387.

[14] D..M. Saul, M. Wortis, D. Stauffer, Phys. Rev. B 9 (1974) 4964.

[15] C. Ekiz, M. Keskin, O. Yalçin, Physica A 293 (2001) 215.

[16] R. Michnas, Physica A 98 (1979) 403.

[17] P.D. Beale, Phys. Rev. B 33 (1986) 1717.

[18] A..M. Ferrenberg, D..P. Landau, Phys. Rev. B 44 (1991) 5081.

[19] C..J. Silva, A. Caparica, J..A. Plascak, Phys. Rev. E 73 (2006) 36702.

[20] J..C. Xavier, F..C. Alcaraz, D. Pena Lara, J..A. Plascak, Phys. Rev. B 57 (1998) 11575.

[21] O..F. Alcantara Bonfim, Physica A 130 (1985) 367.

[22] S. Moss de Oliveira, P..M..C. Oliveira, F..C. SáBarreto, J. Stat. Phys. 78 (1995) 1619.

[23] F..C. Sá Barreto, A..L. Mota, Physica A 391 (2012) 5908.

[24] G..A. Braga, S..J. Ferreira, F..C. Sá Barreto, Braz. J. Phys. 23 (1993) 343.

[25] G..A. Braga, S..J. Ferreira, F..C. Sá Barreto, J. Stat. Phys. 76 (1994) 819.

[26] R. Honmura, T. Kaneyoshi, J. Phys. C: Solid State Phys. 12 (1979) 3979.

[27] J..P. Santos, F..C. Sá Barreto, Physica A 442 (2016) 22.

[28] M. Kerouad, M. Saber, J..W. Tucker, J. Magn. Magn. Mater. 146 (1995) 47.

[29] C. Ekiz, M. Keskin, Phys. Rev. B 66 (2002) 054105.

[30] N..N. Bogoliubov, J. Phys. (USSR) 11 (1947) 23.

[31] R..P. Feynman, Phys. Rev. 97 (1955) 660.

[32] H. Falk, Am. J. Phys. 38 (1970) 858.

[33] H..B. Callen, Phys. Lett. 4 (1963) 161.

[34] K. Kanôand, S. Naya, Prog. Theor. Phys. 10 (1953) 158.

[35] L. Onsager, Phys. Rev. 65 (1944) 117.

[36] H.-O. Deuer, J. Phys. 26 (1993) L333. 